\title{
A psicanálise na desconstrução dos gatilhos para o transtorno de pânico
}

\author{
Psychoanalysis in deconstructing triggers for panic disorder \\ El psicoanálisis en la deconstrucción de los desencadenantes del trastorno de pánico
}

Sara Maria de Brito Sousa Ximenes ORCID: https://orcid.org/0000-0002-9306-602X Cristo Faculdade do Piauí, Brasil E-mail: sarinhaximenes@hotmail.com

Luís Carlos Lopes Barbosa ORCID: https://orcid.org/0000-0002-4272-1290 Cristo Faculdade do Piauí, Brasil

E-mail: carlosloppess@ outlook.com

Nájla Laísa Assunção Rodrigues ORCID: https://orcid.org/0000-0002-1857-1341 Cristo Faculdade do Piauí, Brasil E-mail: najlalar11@gmail.com

Marcos Roberto Nascimento Sousa ORCID: https://orcid.org/0000-0003-1634-5276 Cristo Faculdade do Piauí, Brasil

E-mail: marcosrobertoenfpi@gmail.com

Nayla Letícia Assunção Rodrigues ORCID: https://orcid.org/0000-0002-6847-1269 Instituto Federal do Piauí, Brasil

E-mail: assuncaonayla@gmail.com

Maria Anasha Costa Souza

ORCID: https://orcid.org/0000-0001-5824-0873 Cristo Faculdade do Piauí, Brasil E-mail: anashapiripiri@gmail.com

Suzana Pereira Alves

ORCID: https://orcid.org/0000-0003-1822-9762 Cristo Faculdade do Piauí, Brasil

E-mail: suzaninhaalves10@gmail.com

Glenda Machado de Sampaio ORCID: https://orcid.org/0000-0002-5637-0831 Cristo Faculdade do Piauí, Brasil

E-mail: glenda_sampaio20-11@ hotmail.com

Kennedi Anderson Araújo Dias ORCID: https://orcid.org/0000-0003-4619-5774 Universidade Estadual do Piauí, Brasil

E-mail: Kennedi0704@outlook.com

Luís Henrique Araújo Andrade ORCID: https://orcid.org/0000-0002-3504-0710 Cristo Faculdade do Piauí, Brasil

E-mail: luishenriqueenf22@gmail.com

Iasmim Escórcio de Brito Melo ORCID: https://orcid.org/0000-0002-5984-0309 Cristo Faculdade do Piauí, Brasil E-mail: Iasmimedb@gmail.com

Maria Eduarda Freire Silva ORCID: https://orcid.org/0000-0002-1925-9122 Cristo Faculdade do Piauí, Brasil

E-mail: mariaeduarda1012001@gmail.com Suzana Myrlla Medeiros de Oliveira ORCID: https://orcid.org/0000-0003-0763-3081 Cristo Faculdade do Piauí, Brasil E-mail: suhmyrlla@gmail.com

José Gabriel Fontenele Gomes ORCID: https://orcid.org/0000-0001-6114-0726 Cristo Faculdade do Piauí, Brasil

E-mail: jgabrielfontenele@gmail.com

Anne Heracléia de Brito e Silva ORCID: https://orcid.org/0000-0002-3414-8308 Cristo Faculdade do Piauí, Brasil

E-mail: jgabrielfontenele@gmail.com 


\begin{abstract}
Resumo
Dentre os distúrbios mentais que mais crescem na atualidade, pode-se citar e conceituar que o Transtorno de Pânico (TP) é um transtorno de ansiedade conhecido por seu impacto negativo na qualidade de vida (QV) dos pacientes, independentemente de outros fatores. Mediante o exposto, objetiva-se discorrer, à luz de evidências, como a Psicanálise pode ser utilizada para o Tratamento do Transtorno de Pânico, assim como identificar quais são os gatilhos responsáveis por desenvolver o transtorno mental em questão, e como os profissionais de saúde podem trabalhar ajudando nessa redução e remissão dos casos clínicos associados ao TP. As causas exatas da síndrome do pânico são desconhecidas, embora a Ciência acredite que um conjunto de fatores possa desencadear o desenvolvimento deste transtorno: situações extremas de estresse; crises financeiras; brigas; separações ou mortes na família; experiências traumáticas na infância ou depois de assaltos e sequestros; Realizou-se um estudo bibliográfico utilizando bases de dados eletrônicos Scielo e BVS, com intermédio dos descritores cadastrados em Ciência da SaúdeDeCS: Psicanálise, Transtorno de Pânico, Causas e Profissionais da Saúde. Consistindo de uma observação seletiva e interpretativa dos mesmos, extraindo pontos relevantes, a fim de atribuir a esta pesquisa uma condição explicativa. Assim, conclui-se que é necessário o treinamento dos profissionais de saúde para a abordagem de pessoas com TP. Ademais, deve-se aplicar à população medidas de como reconhecer o que desencadeia suas crises (quais os gatilhos), além de aumentar a presença dos psicólogos tanto hospitalares, quanto clínicos, por meio do NASF nas ESF, promovendo o atendimento biopsicossocial.
\end{abstract}

Palavras-chave: Psicanálise; Transtorno de pânico; Profissionais da saúde.

\begin{abstract}
Among the mental disorders that are growing the most today, it can be mentioned and conceptualized that Panic Disorder (PD) is an anxiety disorder known for its negative impact on patients' quality of life (QOL), regardless of other factors. Based on the above, the objective is to discuss, in the light of evidence, how Psychoanalysis can be used for the Treatment of Panic Disorder, as well as to identify which are the triggers responsible for developing the mental disorder in question, and how health professionals they can work helping to reduce and remit clinical cases associated with PD. The exact causes of panic syndrome are unknown, although Science believes that a set of factors can trigger the development of this disorder: extreme stress situations; financial crises; fights; separations or deaths in the family; traumatic experiences in childhood or after robberies and kidnappings; A bibliographic study was conducted using electronic databases Scielo and VHL, using the descriptors registered in Health Science-DeCS: Psychoanalysis, Panic Disorder, Causes and Health Professionals. Consisting of a selective and interpretive observation of the same, extracting relevant points, in order to give this research an explanatory condition. Thus, it is concluded that it is necessary to train health professionals to approach people with PD. In addition, measures should be applied to the population on how to recognize what triggers their crises (which are the triggers), in addition to increasing the presence of both hospital and clinical psychologists through the NASF in the FHS, promoting biopsychosocial care.
\end{abstract}

Keywords: Psychoanalysis; Panic disorder; Health professionals.

\title{
Resumen
}

Entre los trastornos mentales que más están creciendo en la actualidad, se puede mencionar y conceptualizar que el Trastorno de Pánico (TP) es un trastorno de ansiedad conocido por su impacto negativo en la calidad de vida (CV) de los pacientes, independientemente de otros factores. Con base en lo anterior, el objetivo es discutir, a la luz de la evidencia, cómo se puede utilizar el psicoanálisis para el tratamiento del trastorno de pánico, así como identificar cuáles son los desencadenantes responsables del desarrollo del trastorno mental en cuestión, y cómo la salud profesionales con los que pueden trabajar ayudando a reducir y remitir los casos clínicos asociados a la EP. Se desconocen las causas exactas del síndrome de pánico, aunque Science cree que un conjunto de factores pueden desencadenar el desarrollo de este trastorno: situaciones de estrés extremo; crisis financiera; peleas separaciones o muertes en la familia; experiencias traumáticas en la infancia o después de robos y secuestros; Se realizó un estudio bibliográfico utilizando las bases de datos electrónicas Scielo y VHL, utilizando los descriptores registrados en Ciencias de la Salud-DeCS: Psicoanálisis, Trastorno de Pánico, Causas y Profesionales de la Salud. Consiste en una observación selectiva e interpretativa de los mismos, extrayendo puntos relevantes, con el fin de darle a esta investigación una condición explicativa. Por tanto, se concluye que es necesario formar a los profesionales sanitarios para abordar a las personas con EP. Además, se deben aplicar medidas a la población sobre cómo reconocer qué desencadena sus crisis (cuáles son los desencadenantes), además de incrementar la presencia de psicólogos tanto hospitalarios como clínicos a través de la NASF en la ESF, promoviendo la atención biopsicosocial.

Palabras clave: Psicoanálisis; Trastorno de pánico; Profesionales de la salud.

\section{Introdução}

Os Transtornos Mentais (TM) constituem um problema de saúde pública. Segundo a World Health Organization (2010), cerca de 450 milhões de pessoas sofrem de algum TM, responsável por 8,8\% da mortalidade e 16,6\% de incapacidade 
dentre as doenças em países de baixa e média renda, promovendo, assim, o estudo cada vez mais aprofundado e recorrente dessa série de transtornos.

Dentre os distúrbios mentais que mais crescem na atualidade, pode-se citar e conceituar que o Transtorno de Pânico (TP) é um transtorno de ansiedade conhecido por seu impacto negativo na qualidade de vida (QV) dos pacientes, independentemente de outros fatores (American Psychiatric Association, 2014). E que dentro da garantia do bemestar mental, tem-se técnicas para diminuir e até mesmo cessar os gatilhos promotores da sintomatologia psicossomática associada.

O TP é um dos transtornos de ansiedade, caracterizado por ataques de pânico recorrentes acompanhados por uma persistente preocupação com ataques adicionais e alterações mal adaptativas do comportamento (Associação Americana de Psiquiatria - DSM-V). A assistência ao paciente que sofre de problemas mentais é recorrente e tende a crescer cada vez mais durante e após o período pandêmico que se instala na contemporaneidade (Zuardi, 2017).

No que tange aos acadêmicos, o estudo e a suscitação das temáticas abordadas pela Psicanálise, levam ao interesse em pesquisa científica de um dos maiores nomes dessa corrente psicológica, que é Freud. Assim, trará conhecimentos adquiridos através da leitura de obras consagradas pelo autor e o aprendizado teórico essencial para a prática clínica nos hospitais, mas também na Atenção Básica.

Para a sociedade, é de suma importância o esclarecimento a respeito de um dos transtornos mentais mais evidentes nesse cenário mundial. Assim como a tentativa de desmistificar conceitos e desconstruir preconceitos que possam corroborar ainda mais para a incidência dos gatilhos que desencadeiam o Transtorno de Pânico.

Assim, a Psicanálise foi eleita como teoria central para tentar solucionar os gatilhos responsáveis pelo Transtorno do Pânico em pacientes assistidos por profissionais de Enfermagem, tanto no âmbito hospitalar, como da atenção básica. Mediante o exposto, objetiva-se discorrer, à luz de evidências, como a Psicanálise pode ser utilizada para o Tratamento do Transtorno de Pânico, assim como identificar quais são os gatilhos responsáveis por desenvolver o transtorno mental em questão, e como os profissionais de saúde podem trabalhar ajudando nessa redução e remissão dos casos clínicos associados ao TP.

\section{Metodologia}

O presente estudo teve como proposta de metodologia a revisão bibliográfica simples. Esta contou com a pesquisa, leitura exploratória e seletiva de estudos, artigos originais e de revisão de literatura, com recorte de 2007 a 2020, pesquisados nas bases de dados, Scientific Electronic Library Online (SciELO) e Biblioteca Virtual em Saúde (BVS), assim como dados disponibilizados pelo Ministério da Saúde, em forma de manuais, além de livros consagrados de um dos maiores ícones da Psicanálise que é Freud.

A revisão da literatura narrativa ou tradicional, quando comparada à revisão sistemática, apresenta uma temática mais aberta; dificilmente parte de uma questão específica bem definida, não exigindo um protocolo rígido para sua confecção; a busca das fontes não é pré-determinada e específica, sendo frequentemente menos abrangente. A seleção dos artigos é arbitrária, provendo o autor de informações sujeitas a viés de seleção, com grande interferência da percepção subjetiva (Cordeiro et al., 2007, p. 429).

Dessa forma, realizou-se uma leitura exploratória dos materiais coletados a fim de retirar trechos e informações que validassem os pressupostos levantados, assim como que cumprissem com os objetivos desse estudo. A escolha da fundamentação teórica foi baseada em conceitos do TP e da Psicanálise, assim como da pesquisa sobre as principais causas relacionadas ao transtorno e que poderiam funcionar como gatilhos para novas crises. Ademais, buscou-se métodos da Psicanálise como base para uma assistência de Enfermagem significativa durante esses casos. 


\section{Resultados e Discussão}

\subsection{O pânico}

Freud, em 1926, descreveu e caracterizou o Ataque de Ansiedade pode ser provocado por uma afetação que começa bruscamente, repentinamente, na consciência sem ser despertada pelo curso da imaginação. Assim, as pessoas que sofrem do Transtorno de Pânico estão sujeitas a crises de medo agudo de modo recorrente e inesperado.

Além disso, as crises são seguidas de preocupação persistente com a possibilidade de ter novos ataques seguidos de consequências, seja para dificultar a rotina do dia a dia, seja por medo de perder o controle, enlouquecer ou até mesmo ter um ataque no coração, nas palavras de Bernik;

Bastam 30 segundos para o paciente, que estava se sentindo bem, ser tomado inexplicavelmente por sintomas que, de certa forma, todos conhecemos: boca seca, tremores, taquicardia, falta de ar, mal-estar na barriga ou no peito, sufocamento, tonturas. Muitas vezes, tudo isso vem acompanhado da sensação de que algo trágico, como morte súbita ou enlouquecimento, está por acontecer. Nesses casos, é comum a pessoa ter uma reação comportamental de pânico e sair à procura de socorro. Aliás, a sala de espera dos prontos-socorros é um dos lugares onde o médico mais se depara com transtornos de pânico (Bernik, 2012).

Dessa forma o indivíduo quando está em seu momento de crise acaba não tendo consciência plena das suas ações, podendo ser mudadas de acordo com o ambiente na qual ele esteja no momento, seja esse em casa ou até mesmo já no próprio PS. Esse mal-estar radical que a pessoa sente percorrer por entre suas veias, acaba trazendo sinal de alerta ao mesmo onde ele se encontra hipervigilante a todo instante. Não há como o paciente prever as crises de pânico, pelo menos não nos estágios iniciais desse transtorno, pois parece não haver nada específico capaz de desencadear o ataque. Mas há indícios e pesquisas que apontam que lembrar-se de ataques de pânico anteriores possam contribuir e chegar a levar a uma nova crise.

Segundo a tabela de Zuardi, observa-se os principais sintomas relacionados ao Transtorno de Pânico segundo o DSMV e também já correlacionados por Freud em seus estudos. 
Quadro 1 - Ataques de pânico: comparação entre os critérios do DSM-V e a descrição de Freud.

\begin{tabular}{|c|c|}
\hline DSM-V & Freud \\
\hline $\begin{array}{l}\text { Surgimento abrupto de medo e desconforto } \\
\text { intensos, que atingem um pico em alguns minutos e } \\
\text { durante os quais ocorrem quatro ou mais dos } \\
\text { sintomas abaixo: } \\
\text { - Palpitações, coração pulsando forte ou } \\
\text { - Acelerado } \\
\text { - Sudorese } \\
\text { - Tremor } \\
\text { - Sensação de falta de ar ou de fôlego } \\
\text { - Sensação de desmaio } \\
\text { - Náusea ou desconforto abdominal } \\
\text { - Parestesia } \\
\text { - Dor ou desconforto no peito } \\
\text { - Calafrios ou sensação de calor } \\
\text { - Desrealização (sentimentos de irrealidade) } \\
\text { - Despersonalização (sentindo-se fora de si } \\
\text { - mesmo) } \\
\text { - Medo de perder o controle ou enlouquecer } \\
\text { - }\end{array}$ & $\begin{array}{l}\text { (Ansiedade...) Pode irromper, de repente, na consciência sem } \\
\text { ser despertada pelo curso da imaginação e provocar assim, } \\
\text { um Ataque de Ansiedade... Eis uma relação das formas do } \\
\text { Ataque de Ansiedade } \\
\text { - Palpitações, arritmias breves e } \\
\text { - Taquicardia } \\
\text { - Ataques de suor } \\
\text { - Ataques de tremores } \\
\text { - Dispneia nervosa } \\
\text { - Convulsões, fáceis de confundir com ataques } \\
\text { histéricos } \\
\text { - Diarreias emergentes }\end{array}$ \\
\hline
\end{tabular}

Fonte: Zuardi (p. 58, 2017).

Através da tabela, observa-se que mesmo perpassando tempos e estudos diversos, há consenso sobre a apresentação de alguns sintomas, como as palpitações, arritmias e taquicardias, o suor e os tremores, e o medo frequente de estar desenvolvendo um infarto ou de estar morrendo, o que leva o paciente a recorrer a um PS para ouvir que todos os seus sinais vitais estão dentro da normalidade e que os sintomas nada mais são que frutos de um sofrimento psíquico relacionado ao Transtorno de Pânico.

Temos no caso do TP um exemplo da ausência de fronteiras entre o somático e o psíquico, pois a mistura dos sintomas físicos e emocionais podem confundir o processo diagnóstico. O profissional atua em um contexto desafiador, pois se vê em meio a demandas que, muitas vezes, incluem questões de outra ordem além da orgânica. É fundamental que apure sua escuta para realizar um diagnóstico diferencial e o encaminhamento adequado, devendo ter cuidado ao se comunicar com o paciente que busca atendimento (Nogueira, 2018).

\subsection{Os Gatilhos para o Ataque de pânico}

As causas exatas da síndrome do pânico são desconhecidas, embora a Ciência acredite que um conjunto de fatores possa desencadear o desenvolvimento deste transtorno, sendo as situações extremas de estresse, crises financeiras, brigas, separações ou morte de familiares, experiências traumáticas na infância ou depois de sequestros ou assaltos, bem como pessoas cujos pais possuem transtornos de ansiedade possuem maior susceptibilidade de desenvolver o transtorno (Masci, 2020). 
De acordo com alguns estudos publicados pela Revista Latinoamericana de Psicopatologia Fundamental com pacientes na qual possuem Transtorno de Pânico indicam que a resposta natural do corpo a situações de perigo esteja diretamente envolvida nas crises de pânico que o indivíduo vem a ter. Apesar disso, ainda não está claro por que esses ataques acontecem em situações nas quais não há qualquer evidência de perigo iminente. Em outro lugar, Strachey afirma que prefere o termo "ansiedade" (anxiety) para se referir "à vivência de sofrimento psíquico determinado pela presença de um conflito interno" (Klein, 2017, p. 457).

Partindo dessa afirmação de Strachey, é perceptível que o paciente quando tem não só o Transtorno de Pânico como a ansiedade, suas crises acabam se tornando mais elevadas e bruscas, fazendo com que eles acabem nem entendendo o que se passa em si mesmo. O Manual Diagnóstico e Estatístico de transtornos mentais (2014), considera que para caracterizar o ataque de Pânico o paciente precisa ter um surto abrupto de medo ou desconforto que alcança pico em minutos e durante o qual ocorrem quatro ou mais sintomas de uma lista de 13 sintomas físicos e cognitivos.

\subsection{A Psicanálise}

Desenvolvida por Sigmund Freud, a Psicanálise consiste em um método com técnicas, bem como um conjunto de teorias que podem ser aplicadas nos processos de estudos do inconsciente (Mendes, 2020). Dessa forma, compreende-se que essa linha de pensamento trabalha aquilo que está por trás do que o ser humano é capaz de enxergar sobre si mesmo e suas questões internas.

É partindo da lógica do inconsciente, ao qual dá grande destaque, que a Psicanálise inaugura uma maneira peculiar de compreensão dos conceitos de saúde e doença, subvertendo a lógica das polaridades ao não estabelecer uma hierarquia de critérios que delimite o que é saudável e o que é doente. Mais do que isso, busca questionar os fundamentos dessa organização e a constituição da subjetividade (Silva, 2009). Ou seja, apreende-se que, no lugar de buscar o restabelecimento da saúde perfeita, a Psicanálise pretende questionar as manifestações orgânicas e o sofrimento, porque através deles o sujeito reconhece os afetos e a construção subjetiva da sua própria imagem corporal (Pereira, 1999).

Diante dos fenômenos psicossomáticos, a Psicanálise precisou lidar com uma nova realidade clínica. Ao lado da neurose e da psicose, novas manifestações clínicas demandaram do psicanalista uma adaptação técnica. As manifestações somáticas e pré-simbólicas se aproximam dos actings, dando origem ao adoecimento psicossomático não simbolizado e ao surgimento do sintoma (Ferraz, 2007). Dentre essa somatização, considera-se a que leva aos sintomas do TP, que recentemente foi desmembrado dos Transtornos de Ansiedade, gerando seu próprio CID.

Visto isso, essa corrente contribui para a compreensão de inúmeros distúrbios associados a fatores psicossomáticos que estão situados no inconsciente, fazendo parte da realidade de cada um dos indivíduos e se manifestando de maneira peculiar. Contudo, alguns pacientes podem relatar sintomas corporais em comum, como: taquicardia, falta de ar, desmaios etc. Ademais, até mesmo sentimentos mais complexos e de difícil compreensão (Rodrigues, 2020).

Como exemplo de um desses transtornos psíquicos, é possível mencionar o Pânico, que consiste em uma patologia muito recorrente em indivíduos no mundo inteiro, conforme já citado anteriormente, no qual os acometidos são afetados por uma sintomatologia psicossomática. Dessa forma, a Psicanálise pode ser um instrumento de grande relevância na desconstrução dos gatilhos desse mal.

\subsection{A Psicanálise como aliada no tratamento do Transtorno de Pânico}

Com relação ao tratamento, recomenda-se a combinação do tratamento medicamentoso aliado com a terapia. O tratamento para ser ideal deverá ocorrer por um ano. A melhora costuma ocorrer entre duas e quatro semanas, porém segundo 
Bernik (2012), muitos pacientes desistem do tratamento, pelos efeitos colaterais. Dessa forma, a associação da terapia Psicanalista, ajuda o paciente na compreensão de suas questões internas causadoras do transtorno estudado.

De modo geral, combinar as terapias tem sido uma escolha frequente de terapeutas e pacientes quando tem disponibilidade. Mostrou que podem proporcionar um controle imediato das angústias, reduzir as recaídas e diminuir o uso de medicamento a largo prazo (American Psychiatric Association, 2010). Além de ser adequado ao tipo de sintomas e queixas de cada paciente, em sua singularidade de apresentação da doença.

Pode haver muitas semelhanças entre os pacientes, mas é importante diferenciar as individualidades de cada um. A frequência com que as crises aparecem nos pacientes são muito variáveis. Ter sensibilidade com estas diferenças e fazer com que o paciente entenda estes sintomas e até monitorar diariamente os mesmos são formas de tentar revelar gatilhos de sintomas e ataques de pânico. Outra vez é importante afastar qualquer outro distúrbio psiquiátrico para obter melhor resultado com a terapêutica (Honda, 2020, p. 15).

O paciente deve aprender a identificar os gatilhos e saber as sensações físicas específicas que se manifestam durante o ataque de Pânico, e o tratamento psicoterápico pode ser realizado individualizado ou em grupo, e segundo o autor as sessões têm como características serem estruturada, breves e eficazes (Baère, 2015). Logo, os profissionais da saúde conseguem explicar o prognóstico positivo a ser encontrado com o seguimento dos protocolos de cuidados adequados ao TP.

\section{Conclusão}

Determina-se, pois, que a Psicanálise pode ser aliada no tratamento do TP a partir do momento que o paciente é levado a observar-se e autoconhecer-se por meio da introspecção- uma viagem a seu mundo interno, visando buscar a causa efetiva das perturbações recorrentes e tratá-las. A Psicanálise de Freud utiliza-se até mesmo dos sonhos como representação daquilo que não é facilmente visível e compreensível aos portadores do transtorno. Dessa forma, explicitar tais técnicas, além da terapia facilita o paciente a compreender que questões pessoais como brigas, estresse e/ou traumas de abuso podem ser gatilhos para a psicossomatização observada no TP.

Conclui-se que é necessário o treinamento dos profissionais de saúde para a abordagem de pessoas com TP, pois só assim terão pessoas mais sensíveis aos transtornos mentais que chegam às urgências e emergências hospitalares. Ademais, deve-se aplicar à população medidas de como reconhecer o que desencadeia suas crises (quais os gatilhos), além de aumentar a presença dos psicólogos tanto hospitalares, quanto clínicos, por meio do NASF nas ESF.

Dessa forma, acredita-se que possam ser diminuídos os casos de TP que chegam a âmbito hospitalar, mas também, que a porta de entrada do sistema ofereça uma abordagem mais holística e biopsicossocial à população. Além de promover a valorização da saúde mental como requisito de uma qualidade de vida satisfatória nos conceitos seguidos pela OMS e MS do Brasil.

\section{Referências}

American Psychiatric Association. (2010). Practice Guideline For The Treatment of Patients With Panic Disorder. https://psychiatryonlineorg/pb/assets/raw/sitewide/practice_guidelines/guidelines/panicdisorderpdf

American Psychiatric Association. (2014). Manual Diagnóstico E Estatístico De Transtornos Mentais (DSM-5). (5a ed.). Artmed.

Baère, T. D. (2015). Técnicas cognitivas-comportamentais para o tratamento do transtorno do pânico. Psicologia PT, 1(1), 1-9. https://www.psicologia.pt/artigos/ver_artigo.php?codigo=A0937

Bernik, M. (2012). Síndrome do pânico | Entrevista. [Weblog]. https://drauziovarella.uol.com.br/entrevistas-2/sindrome-do-panico-entrevista/

Cordeiro, A. M. et al. (2007). Revisão sistemática: uma revisão narrativa. Rev. Col. Bras. Cir., 34(6), 428-431. https://www.scielo.br/scielo.php?sc ript=sci_arttext\&pid=S0100-69912007000600012 
Research, Society and Development, v. 10, n. 3, e31010313265, 2021

(CC BY 4.0) | ISSN 2525-3409 | DOI: http://dx.doi.org/10.33448/rsd-v10i3.13265

Ferraz, F. C. (2007). A tortuosa trajetória do corpo na psicanálise. Revista Brasileira de Psicanálise, 41(4), 66-76. http://pepsic.bvsalud.org/scielo.php?script=sci_arttext\&pid=S0486-641X2007000400007

Honda, R. W. (2020). Transtorno de pânico: uma abordagem homeopática. Relato de Caso. Homeoindex-Homeopatia, 1(1), 1-45. https://pesquisa.bvsalud.org/portal/resource/pt/biblio-1122974

Klein, T. \& Herzog, R. (2017). Inibição, sintoma e medo? Algumas notas sobre Angst na psicanálise. Rev. Latinoam. Psicopatol. Fundam, 20(4), 686-704. https://www.scielo.br/scielo.php?pid=S1415-47142017000400686\&script=sci_abstract\&tlng=pt

Masci, C. (2020). Síndrome do pânico: o que é, sintomas e tratamento. Minha Vida. https://www.minhavida.com.br/saude/temas/sindrome-do-panico

Mendes, R. C. C. S. \& Paravidini, J.L.L. (2020). Psicanálise em pesquisas: o método investigativo e algumas afirmações essenciais a duas críticas recorrentes. Ágora, 23(3), 47-53. https://www.scielo.br/scielo.php?script=sci_arttext\&pid=S1516-14982020000300047

Nogueira, J. F. O., Neves, R. T., Ferreira. A. P. C., Medeiros, A. A., Carbonari, K. F. B. S. F. (2018). Transtorno do pânico: cardiologia e psicologia em ação. Rev. Soc. Cardiol., 28(3). https://pesquisa.bvsalud.org/portal/resource/pt/biblio-964378

Pereira, M. E. C. (1999). Pânico e desamparo. Editora Escuta.

Rodrigues, M. S. \& Munoz, N. M. (2020). Entre angústia e ato: desafios para o manejo da urgência subjetiva na clínica psicanalítica. Ágora, 23(3), 90-98. https://www.scielo.br/scielo.php?pid=S1516-14982020000300090\&script=sci_abstract\&tlng=pt

Silva, M. M. (2009). Para além da saúde e da doença: O caminho de Freud. Ágora, 12(2), 259-274. https://www.scielo.br/scielo.php?pid=S151614982009000200007\&script=sci_abstract\&tlng=pt\#: :text=ISSN\%201809\%2D4414.,\%C3\%A9\%20pensada\%20pela\%20sa\%C3\%BAde\%20mental.

World Health Organization. (2010). People with mental disabilities cannot be forgotten. World Health Organization. https://www.who.int/mediacentr e/news/releases/2010/mental_disabilities_20100916/en/

Zuardi, A. W. (2017). Características básicas do transtorno do pânico. Medicina (Ribeirão Preto, Online), 50(1), 56-63. https://www.revistas.usp.br/rmrp/article/view/127539 